\title{
Very high energy $\gamma$ ray absorption by the galactic interstellar radiation field (Research Note)
}

\author{
J.-L. Zhang, X.-J. Bi, and H.-B. Hu
}

Key laboratory of particle astrophysics, Institute of High Energy Physics, Chinese Academy of Sciences, PO Box 918-3, Beijing 100049, PR China

Received 26 October 2005 / Accepted 14 December 2005

ABSTRACT

Aims. We calculate the attenuation of very high energy $\gamma$ rays from Galactic sources.

Methods. A recent calculation of the Galactic interstellar radiation field shows that the infra-red radiation background near the Galactic Center is very intense. The attenuation of very high energy $\gamma$ rays by this radiation is strong and occurs at lower energies than previously thought. Results. Our result shows that the cutoff of the high energy $\gamma$ ray spectrum begins at about $20 \mathrm{TeV}$ and reaches about $10 \%$ for $50 \mathrm{TeV} \gamma$ rays.

Key words. ISM: cosmic rays - infrared: ISM - gamma rays: observations

\section{Introduction}

The Galactic interstellar radiation field (ISRF) is very important in understanding the spectrum of the very high energy (VHE) $\gamma$ rays originating from galactic sources. It is generally believed that at the acceleration site the VHE cosmic ray electrons interact with ISRF photons via inverse Compton scattering, which generates VHE $\gamma$ rays. On the way to the observer, the VHE $\gamma$ ray could be absorbed due to its interaction with the ISRF that changes its spectrum.

Previous studies of the absorption of VHE $\gamma$ rays have been focused on those from high redshift sources which interact with the extragalactic photon background. The absorption of VHE $\gamma$ rays within the Galaxy is believed to be small since the $\gamma$ rays travel a short distance. However, in the calculation of Porter \& Strong (2005), which has incorporated a large amount of relevant new astronomical information on stellar populations, Galactic structure and interstellar dust, the density of the infra-red background within the Galaxy is enhanced considerably compared to previous models. A very high infrared background near the galactic center is found with this calculation. Therefore, the attenuation of VHE $\gamma$ rays within the Galaxy may increase if the $\gamma$ rays come from the direction of the galactic center. In another work (Porter \& Allard 2005) the mean free path (MFP) of cosmic ray nuclei was calculated with the new results of the ISRF. It is found that the MFP is greatly decreased considering the infra-red background near the galactic center. In this paper, we estimate the absorption of VHE $\gamma$ rays by the ISRF within the galaxy.

\section{Opacity of the Galactic ISRF to VHE $\gamma$ rays}

The ISRF is composed of star light, infra-red radiation and the cosmic microwave background (CMB). The energy density of star light is determined by the distribution and spectrum of each stellar type. The starlight is then absorbed by dust in the interstellar medium and re-emitted in the infra-red, the density of which depends on the distribution of the dust. The starlight is also scattered by the dust and forms the diffuse galactic light. Starlight dominates the ISRF from $0.1 \mu \mathrm{m}$ to $10 \mu \mathrm{m}$. The emission from very small dust grains dominates from $10 \mu \mathrm{m}$ to $30 \mu \mathrm{m}$ while the emission from larger dust grains with temperatures of about $20 \mathrm{~K}$ dominates from $20 \mu \mathrm{m}$ to $300 \mu \mathrm{m}$. The CMB contributes mainly to emission above $1000 \mu \mathrm{m}$.

A detailed calculation of the ISRF has been established and adopted widely (Strong et al. 2000). Taking into account the new results of the Galactic stellar distribution, dust distribution and other astronomical information, the calculation of the ISRF spectra has been recently updated (Porter \& Strong 2005). Together with the very successful infrared surveys carried out by the Infrared Astronomical Satellite (IRAS) and the Diffuse Infrared Background Experiment (DIRBE) on board the Cosmic Ray Background Explorer (COBE) satellite, our knowledge of the energy (number) density of ISRF photons has greatly improved. The new calculations of the local radiation field show fair agreement with these observations.

Due to the ISRF background, VHE $\gamma$-rays are attenuated when penetrating the radiation background through $\mathrm{e}^{+} \mathrm{e}^{-}$pair 
production when colliding with the ISRF. The $\gamma$-ray spectrum $F_{0}(E)$ at the source is attenuated to

$$
F(E)=F_{0}(E) \mathrm{e}^{-\tau(E, L)},
$$

where $F(E)$ represents the observed spectrum after the attenuation; $\tau(E, L)$ represents the optical depth of the $\gamma$-rays, being a function of the $\gamma$-ray energy and the distance from the $\gamma$-ray source. $\tau(E, L)$ is given as

$$
\begin{aligned}
\tau(E, L)=\int \mathrm{d} \cos (\theta) & \int \mathrm{d} E_{\mathrm{ISRF}} \\
& \times \int_{\text {l.o.s. }} \mathrm{d} L \frac{\mathrm{d} n\left(r, E_{\mathrm{ISRF}}\right)}{\mathrm{d} E_{\mathrm{ISRF}}} \sigma_{\gamma \gamma \rightarrow \mathrm{e}^{+} \mathrm{e}^{-}}(s),
\end{aligned}
$$

where the integration of $\mathrm{d} L$ is along the line of sight of the incoming $\gamma$-rays, $\frac{\mathrm{d} n\left(r, E_{\text {ISRF }}\right)}{\mathrm{d} E_{\text {ISRF }}}$ is the number density of the ISRF photons at the radius $r$ from the GC at energy $E_{\mathrm{ISRF}}, \sigma_{\gamma \gamma \rightarrow \mathrm{e}^{+} \mathrm{e}^{-}}(s)$ is the cross section of the pair production. The cross section is zero below the threshold when the center-of-mass energy $\sqrt{s}<2 m_{\mathrm{e}}$. Above the threshold, the cross section is given by

$$
\begin{aligned}
\sigma_{\gamma \gamma \rightarrow \mathrm{e}^{+} \mathrm{e}^{-}}(s)= & \sigma_{\mathrm{T}} \\
\cdot & \frac{3 m_{\mathrm{e}}^{2}}{2 s} \cdot\left[-\frac{p}{E}\left(1+\frac{4 m_{\mathrm{e}}^{2}}{s}\right)\right. \\
& \left.+\left(1+\frac{4 m_{\mathrm{e}}^{2}}{s}\left(1-\frac{2 m_{\mathrm{e}}^{2}}{s}\right)\right) \log \frac{(E+p)^{2}}{m_{\mathrm{e}}^{2}}\right],
\end{aligned}
$$

where $\sigma_{\mathrm{T}}=8 \pi^{2} \alpha^{2} / 3 m_{\mathrm{e}}^{2}$ is the Thomson cross section for photon elastic scattering on a rest electron, $E=\sqrt{s} / 2$ and $p=\sqrt{E^{2}-m_{\mathrm{e}}^{2}}$ are the energy and the magnitude of momentum of the electron at the center-of-mass system. In the laboratory system, $s$ is given by $s=2 E_{\gamma} E_{\mathrm{ISRF}}(1-\cos \theta)$ with $\theta$ being the angle between the momentum of the incoming $\gamma$ ray and the ISRF photon. For the ISRF photon we have assumed an isotropic distribution when integrating over $\theta$.

The attenuation of extragalactic $\gamma$ rays due to collisions with the CMB from high redshift sources has been studied extensively. The attenuation length can fall below $10 \mathrm{kpc}$ when the $\gamma$ ray energy reaches about $500 \mathrm{TeV}$. As the new calculation of ISRF shows that the number density of far-infrared ISRF (20 $\mu \mathrm{m}$ to $300 \mu \mathrm{m}$ ) at the Galactic Center is almost comparable to that of CMB photons, we therefore expect a significant attenuation at a distance of $10 \mathrm{kpc}$ within the Galaxy for $\gamma$ rays with an energy of about $50 \mathrm{TeV}$.

\section{Attenuation of the VHE $\gamma$ rays from Sgr $A^{*}$}

As the ISRF is most intense at the GC, the $\gamma$ rays passing through the GC should experience the greatest attenuation. Among the limited number of $\mathrm{TeV} \gamma$ ray sources that have been observed in the Milky Way, Sgr A* is the most suitable one for study of this absorption. Sgr A* lies at the GC, about $8 \mathrm{kpc}$ from the Earth. Although its integrated flux above $165 \mathrm{GeV}$ is only $5 \%$ of that of Crab measured by HESS, its energy spectrum is much harder, with a power law index at about 2.2 (Aharonian et al. 2004), which makes its integrated flux above $50 \mathrm{TeV}$ almost comparable to that of the Crab and enables future high statistical power observations.

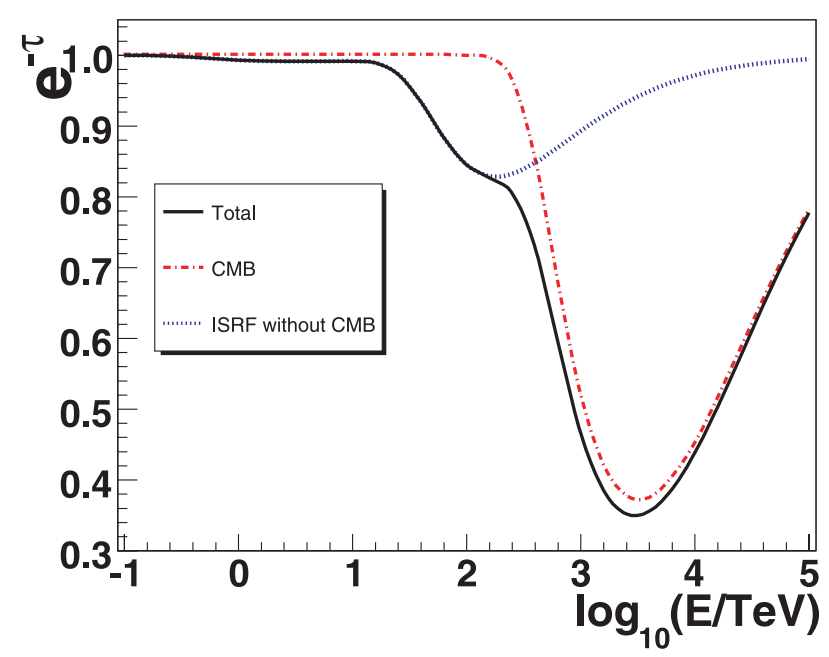

Fig. 1. Attenuation of the VHE $\gamma$ rays due to interaction with the ISRF as a function of the $\gamma$ ray energy. Contributions to the attenuation due to $\mathrm{CMB}$ and the ISRF without CMB are plotted separately.

To calculate the attenuation of the $\gamma$ rays emitted from Sgr A*, we need to know the radial distribution of the number densities of the ISRF photons between the GC and the Earth. As the number density of the ISRF photons falls from the GC to the edge of the Galaxy in the form of an exponential function with a radial scale length of about $4 \mathrm{kpc}$ (Strong et al. 2000 ; Porter \& Strong 2005), $\mathrm{d} n\left(r, E_{\mathrm{ISRF}}\right) / \mathrm{d} E_{\mathrm{ISRF}}$ is interpolated using the densities calculated by Porter and Strong (Porter \& Strong 2005) for the in-plane region at $R=0 \mathrm{kpc}, 4 \mathrm{kpc}$ and $8 \mathrm{kpc}$. Considering that the dust emission might have a different spatial dependence than the overall ISRF spectrum, a linear interpolation has also been examined. There is no difference in attenuation by exponential or linear interpolation.

Figure 1 shows the calculated attenuation for the $\gamma$ rays from $\mathrm{Sgr} \mathrm{A}^{*}$ as a function of $\gamma$ ray energy. The cutoff begins at about $20 \mathrm{TeV}$ and about $10 \%$ is absorbed for $\gamma$ rays at $50 \mathrm{TeV}$ and $20 \%$ for $\gamma$ rays at $100 \mathrm{TeV}$, which is not far from our expectation. Attenuation due to the CMB and the infra-red components is also plotted to compare their contributions. From Fig. 1 it seems that the effects of VHE $\gamma$ ray attenuation should appear if the observation reaches about $50 \mathrm{TeV}$.

\section{Discussion}

Using the recently calculated ISRF distribution, and taking Sgr A* as an example, this work shows that the ISRF may have observable attenuation effects on the spectrum of VHE $\gamma$ rays from galactic sources. As the model might underestimate the ISRF component between 20 to $40 \mu \mathrm{m}$ compared to the observation by FIRAS (Finkbeiner et al. 1999), the real effect could be even larger and start to appear at even lower energies. Observation of the cutoff energy will provide independent information to test and constrain the ISRF model. The recent discovery of new $\mathrm{TeV} \gamma$ ray sources on the galactic plane (Aharonian et al. 2005) will provide more candidates to study the distance dependent cutoff effect.

The attenuation cutoff can be attributed either to an intrinsic cutoff, such as the cutoff spectrum of the injecting 
electron at the $\gamma$ ray source, or to the ISRF absorption discussed here. Therefore a multi-band observation of the $\gamma$ ray source and careful analysis are necessary to reveal the individual contribution, as the intrinsic cutoff can be traced to lower energy bands while absorption takes place only for the high energy bands.

Acknowledgements. This work is supported by the NSF of China under the grant Nos. 10575111, 10105004, 10120130794, and this work is also supported in part by Chinese Academy of Sciences in China.

\section{References}

Aharonian, F., Akhperjanian, A. G., Aye, K.-M., et al. 2004, A\&A, 425, L13

Aharonian, F., Akhperjanian, A. G., Aye, K.-M., et al. 2005, Science, 307, 1938

Finkbeiner, D., Davis, M., \& Schlegel, D. J. 1999, ApJ, 524, 867

Porter, T. A., \& Allard, D. 2005 [arXiv:astro-ph/0507121]

Porter, T. A., \& Strong, A. W. 2005 [arXiv: astro-ph/0507119]

Strong, A. W., Moskalenko, I. V., \& Reimer, O. 2000, ApJ, 537, 763 